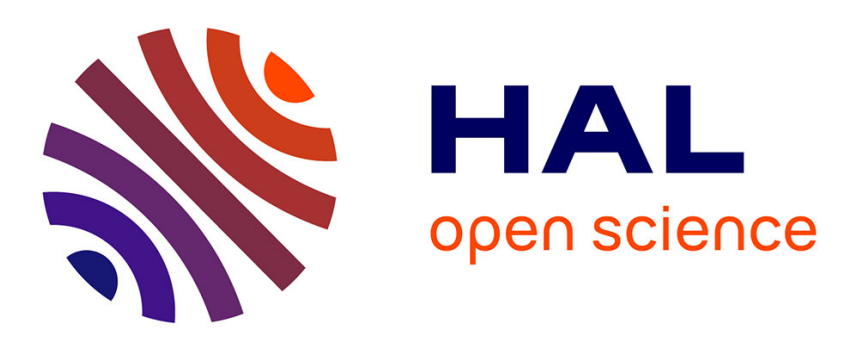

\title{
La prévision des précipitations par recherche d'analogues : état de l'art et perspectives
}

\author{
A. Ben Daoud, Eric Sauquet, M. Lang, C. Obled, G. Bontron
}

\section{To cite this version:}

A. Ben Daoud, Eric Sauquet, M. Lang, C. Obled, G. Bontron. La prévision des précipitations par recherche d'analogues: état de l'art et perspectives. La Houille Blanche - Revue internationale de l'eau, 2009, 6, p. 60 - p. 65. 10.1051/lhb/2009079 . hal-00489961

\section{HAL Id: hal-00489961 https://hal.science/hal-00489961}

Submitted on 7 Jun 2010

HAL is a multi-disciplinary open access archive for the deposit and dissemination of scientific research documents, whether they are published or not. The documents may come from teaching and research institutions in France or abroad, or from public or private research centers.
L'archive ouverte pluridisciplinaire HAL, est destinée au dépôt et à la diffusion de documents scientifiques de niveau recherche, publiés ou non, émanant des établissements d'enseignement et de recherche français ou étrangers, des laboratoires publics ou privés. 


\section{La prévision des précipitations par recherche d'analogues : état de l'art et perspectives}

\section{Precipitation forecasting through an analog sorting technique : state of the art and further investigations}

AURÉLIEN BEN DAOUD, ERIC SAUQUET, MICHEL LANG

Cemagref - Unité de Recherche Hydrologie-Hydraulique

3 bis Quai Chauveau, CP 220, 69336 Lyon, Cedex 9, France

Tél : +33 (0)4 722087 64, Fax : +33 (0)4 7847 78 75, e-mail : aurelien.bendaoud@cemagref.fr

CHARLES OBLED

Laboratoire d'étude des Transferts en Hydrologie et Environnement

Institut National Polytechnique de Grenoble, B.P. 53, 38041 Grenoble, Cedex 9, France

Tél : +33 (0)4 768250 53, Fax : +33(0)4 768250 14, e-mail : charles.obled@hmg.inpg.fr

\section{GUILLAUME BONTRON}

Compagnie Nationale du Rhône

Direction de l'Ingénierie - Eau et Environnement, 2 rue André Bonin, 69316 Lyon, Cedex 04, France

Tél : +33 (0)4 720061 93, Fax : +33 (0)4 721066 62, e-mail : G.Bontron@cnr.tm.fr

a prévision des précipitations par analogie, adaptée des sorties de modèles numériques de prévision, s'est améliorée ces dix dernières années et est actuellement implémentée opérationnellement dans diverses infrastructures françaises. Le premier objectif de cette étude est de dresser l'état de l'art de cette approche. L'application de cette méthode nécessite une base de données contenant les champs des variables qui permettent de caractériser les situations météorologiques passées. Etant donné qu'il existe deux archives de génération différente (réanalyses ERA-40 et NCEP/NCAR), la sensibilité de cette méthode de prévision au choix de l'archive a été étudiée. Les résultats révèlent une faible sensibilité, même si de manière générale les performances sont légèrement supérieures avec l'archive ERA-40, notamment pour les événements pluvieux extrêmes. Enfin, des perspectives d'amélioration de la méthode susceptibles d'être exploitées à court terme sont évoquées.

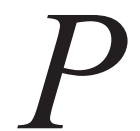

recipitation forecasting based on an adaptation of model outputs through an analog sorting technique has been improved for around ten years. The method runs operationally in several French institutions. First, this short paper presents the state of the art of this approach and the more recent developments. Second, a sensitivity analysis to the choice of the database from which the variables that characterise the past meteorological situations are extracted is performed. Two available archives are tested (ERA-40 and NCEP/NCAR re-analyses). The results show that despite the performances obtained with the ERA-40 database are slightly better, especially for heavy rainfall events, the sensitivity is weak. Finally, further ways for improvement that could be investigated are suggested.

\section{I 口 INTRODUCTION}

Malgré le progrès constant des modèles numériques de prévision du temps (MNP), la quantité de précipitation reste une variable extrêmement difficile à prévoir plusieurs jours à l'avance. En effet, la description exhaustive des processus à l'origine de la pluie est loin d'être connue, puisque de multiples paramètres entrent en jeu dans la formation des nuages et des précipitations (quantité de vapeur d'eau présente dans l'atmosphère, niveaux de saturation et de condensation, vents verticaux, relief, etc.).
Avec la constante augmentation de la résolution des MNP, la prévision des précipitations est généralement fiable jusqu'à 48 heures à l'avance. Néanmoins pour certaines situations météorologiques, il arrive encore que la prévision fournie par les MNP soit erronée moins de 24 heures avant l'occurrence d'un événement pluvieux.

En revanche, d'autres variables sont mieux prévues par les MNP puisque leur variabilité dans le temps et dans l'espace est décrite par des équations robustes. C'est le cas de la pression atmosphérique, variable clé pour réaliser des prévisions. Les champs de pression atmosphérique permet- 
tent en effet de caractériser la circulation atmosphérique à grande échelle et leur analyse est sans doute la première étape du prévisionniste en météorologie. Avec l'expérience, le prévisionniste associe les champs de pression prévus pour les journées à venir à des situations qu'il a déjà rencontrées auparavant. S'il se souvient du temps qu'il a fait lors de ces journées passées au-dessus de la zone de prévision, il aura appliqué de manière instinctive le principe d'analogie entre situations météorologiques.

Plus formellement, le concept d'analogie en météorologie a été introduit par Lorenz [1]. Il repose sur l'hypothèse que deux situations synoptiques analogues doivent engendrer des effets locaux du même ordre. Il peut être utilisé pour corriger les biais des MNP ([2], [3]), étudier les caractéristiques météorologiques d'événements particuliers, ou encore émettre des prévisions probabilistes de précipitations. Cette méthode de prévision des pluies, assimilée à une technique d'adaptation statistique des sorties de MNP et communément appelée " méthode des analogues ", s'appuie notamment sur une archive de variables d'analogie (prédicteurs), c'est-à-dire les variables qui décrivent la situation météorologique et à partir desquelles la similitude entre la situation prévue et une situation passée est évaluée. Depuis quelques années, les réanalyses météorologiques sont utilisées comme archives de variables d'analogie.

Nous dresserons tout d'abord l'état de l'art de la méthode des analogues en donnant l'historique puis en décrivant la dernière version développée par Bontron [4]. Nous évoquerons quelques résultats d'un travail de thèse en cours portant sur la sensibilité de la méthode aux choix de l'archive de réanalyses météorologiques. Enfin, nous évoquerons les perspectives d'amélioration de la méthode qui s'offrent à la prévision opérationnelle des précipitations adaptée par analogie.

\section{II — LA MÉTHODE DE PRÉVISION DES PRÉCIPITATIONS ADAPTÉE PAR ANALOGIE : ÉTAT DE L'ART}

\section{- II.1 BREF HISTORIQUE}

Duband [5] a initié les travaux sur la prévision des précipitations par analogie en France et ses développements ont conduit Electricité De France (EDF) à implanter un système de prévision opérationnel au début des années 1970. Ce système utilisait les champs de pression observés le jour $\mathrm{J}$ et une extrapolation temporelle était effectuée à partir de ces champs prédicteurs pour les jours à venir. Ces champs n'étaient donc pas encore fournis par des MNP. Avec le progrès de ces derniers, la méthode a évolué dans les années 1980 [6] en devenant une méthode d'adaptation statistique des MNP, c'est-à-dire que les champs des variables sur lesquels l'analogie est recherchée étaient désormais prévus par des MNP. La méthode s'est développée ensuite jusque dans les années 1990, avec l'amélioration des prévisions de variables synoptiques. L'échéance de prévision a alors été repoussée à quatre jours. Guilbaud [7] a introduit un nouveau critère d'analogie pour la sélection d'analogues à l'aide de variables définies sur une grille régulière [8]. A partir de ces travaux, Bontron [4] a amélioré les performances de la méthode en hiérarchisant la sélection des situations analogues sur deux niveaux et en utilisant une nouvelle archive de variables d'analogie : les réanalyses météorologiques. Cette mise à jour sera considérée comme un état de référence. Les différentes méthodes ont été implémentées opérationnellement à EDF, à la Compagnie Nationale du Rhône (CNR) en 2005 et dans plusieurs laboratoires. Parallèlement, le concept d'analogie pour la prévision des précipitations a été appliqué dans d'autres pays ([9], [10], [11], [12]).

\section{II.2 DESCRIPTION DE LA MÉTHODE DE RÉFÉRENCE}

De manière générale, la recherche d'analogie repose sur le choix de plusieurs paramètres :

- la variable à prévoir et l'archive historique correspondante (pluviométrique dans notre cas);

- les variables d'analogie qui permettent de caractériser l'état de l'atmosphère et l'archive historique correspondante ;

— le critère qui permet d'identifier une situation analogue ;

- le domaine spatial sur lequel on recherche l'analogie ;

- le nombre de situations analogues à sélectionner.

La méthode dite "de référence », suggérée par Bontron [4], forme le point de départ de travaux de recherche, menés conjointement par la CNR et le Cemagref depuis novembre 2006, en concertation avec le Laboratoire d'étude des Transferts en Hydrologie et Environnement. Ces travaux visent à améliorer la prévision probabiliste de précipitations journalières. Dans la méthode de référence, la sélection de situations analogues à la situation du jour cible (jour C) s'effectue en deux étapes. Les variables, domaines, critères et nombre d'analogues à sélectionner pour chaque niveau d'analogie sont résumés dans le Tableau 1.

Tableau 1 : Paramètres de la sélection de situations analogues à chaque niveau d'analogie [4]

\begin{tabular}{|l|l|l|}
\hline & \multicolumn{1}{|c|}{ Niveau 1 } & \multicolumn{1}{c|}{ Niveau 2 } \\
\hline $\begin{array}{l}\text { Variables } \\
\text { d'analogie }\end{array}$ & $\begin{array}{l}\text { Géopotentiels à } \\
1000 \mathrm{hPa} \text { et } 500 \mathrm{hPa}\end{array}$ & $\begin{array}{l}\text { Humidité relative à } \\
850 \mathrm{hPa} \text { et colonne } \\
\text { totale d'eau }\end{array}$ \\
\hline Domaine & $\begin{array}{l}\text { Echelle synoptique à } \\
\text { optimiser }\end{array}$ & $\begin{array}{l}\text { Echelle locale à } \\
\text { optimiser }\end{array}$ \\
\hline Critère & $\begin{array}{l}\text { Score de Teweles- } \\
\text { Wobus (1954) }\end{array}$ & $\begin{array}{l}\text { Erreur quadratique } \\
\text { moyenne }\end{array}$ \\
\hline $\begin{array}{l}\text { Nombre } \\
\text { d'analogues }\end{array}$ & A optimiser & A optimiser \\
\hline
\end{tabular}

La première sélection (niveau 1) permet de constituer un échantillon de situations similaires à la situation cible à échelle synoptique, en termes de circulation générale. A l'issue de cette étape, N1 situations sont alors retenues. La seconde sélection (niveau 2) permet de ne retenir, parmi les 
situations de l'échantillon du niveau 1, que les situations analogues à la situation du jour $C$ à une échelle plus locale, en termes d'humidité. N2 situations sont finalement retenues comme analogues. Les nombres N1 et N2, fonction notamment de la longueur de l'archive de variables d'analogie, ainsi que les domaines d'analogie doivent être optimisés pour la station ou le bassin versant d'intérêt. Finalement, l'échantillon de dates analogues permet, à l'aide des pluies observées au cours de ces journées, de construire la distribution empirique des pluies prévue pour le jour $C$.

\section{III - SENSIBILITÉ DE LA MÉTHODE DE PRÉVISION AU CHOIX DE L'ARCHIVE DE VARIABLES D'ANALOGIE}

L'archive de prédicteurs utilisée par Bontron [4], issue des réanalyses NCEP/NCAR [13] à résolution $\left(2.5^{\circ} \times 2.5^{\circ}\right)$, est apparue comme plus homogène que celles disponibles auparavant, lesquelles résultaient de l'archivage au fil du temps des analyses produites par les modèles opérationnels de prévision. Cette archive présente également l'intérêt d'inclure de nombreuses variables météorologiques, autres que le géopotentiel, telles que celles utilisées lors du second niveau de la sélection des situations analogues.

Aujourd'hui, deux archives sont disponibles sur des périodes suffisamment longues avec une résolution de 2,5 $:$ les réanalyses ERA-40 (ERA) [14] et les réanalyses NCEP/ NCAR (NNR) [13]. En préalable au travail sur la méthode en elle-même, les variables d'analogie extraites de ces bases de données ont été comparées afin d'apprécier les différences entre les deux réanalyses [15]. Des valeurs aberrantes d'humidité relative à $850 \mathrm{hPa}$ ont été détectées dans l'archive ERA. Certaines valeurs sont inférieures à $0 \%$ et une proportion non négligeable est supérieure à $100 \%$. Une tentative de correction de ces valeurs a ensuite été menée mais elle n'a pas été concluante.

Une analyse de la sensibilité de la méthode de référence au choix de l'archive de variables d'analogie a été effectuée sur le bassin versant de la Saône, dont les pluies de bassin ont été extraites des réanalyses SAFRAN [16]. Cette analyse a été réalisée dans un contexte de prévision parfaite, c'est-à-dire que les situations pour lesquelles on émet une prévision ne sont pas prévues par un MNP, mais tirées des archives de réanalyses. La période de situations cibles et candidates s'étend du 01/09/1972 au 30/08/2002 (soit 10987 dates).

\section{III.1 OPTIMISATION DES NOMBRES D'ANALOGUES À SÉLECTIONNER À L'AIDE DU CRPS}

La sensibilité de la méthode au choix de l'archive de variables d'analogie est d'abord étudiée en optimisant les nombres $\mathrm{N} 1$ et $\mathrm{N} 2$ d'analogues sélectionnées aux niveaux 1 et 2. Ils sont optimisés à l'aide du score Continuous Ranked Probability Score (CRPS) [17]. La performance de prévision est finalement exprimée en normalisant le CRPS par rapport à la climatologie. Le score résultant, nommé RSS (Relative
Skill Score) (1), a pour valeur 1 pour une prévision parfaite, 0 pour une prévision équivalente à la prévision climatologique, et est négatif pour une prévision de moins bonne qualité que la prévision climatologique :

$$
R S S=\frac{C R P S_{M}-C R P S_{c l i m}}{C R P S_{p a r f}-C R P S_{c l i m}}
$$

où $C R P S_{M}$ est le score calculé avec la méthode à évaluer, $C R P S_{c \lim }$ est le score calculé avec la prévision climatologique, et $C R P S_{\text {parf }}$ est le score parfait (valeur nulle).

Ce score a été calculé pour chaque couple $(\mathrm{N} 1, \mathrm{~N} 2)$. Le nombre N1 optimal est de 70 (resp. 80) lorsque l'archive ERA (resp. NNR) est utilisée. La Figure 1 montre l'évolution du RSS en fonction de N2 pour le nombre N1 optimal associé à chaque archive (ERA N2 et NNR N2). L'évolution du RSS en fonction de N1 (niveau 1 uniquement appliqué) a également été tracée pour chacune des archives (ERA N1 et NNR N1).

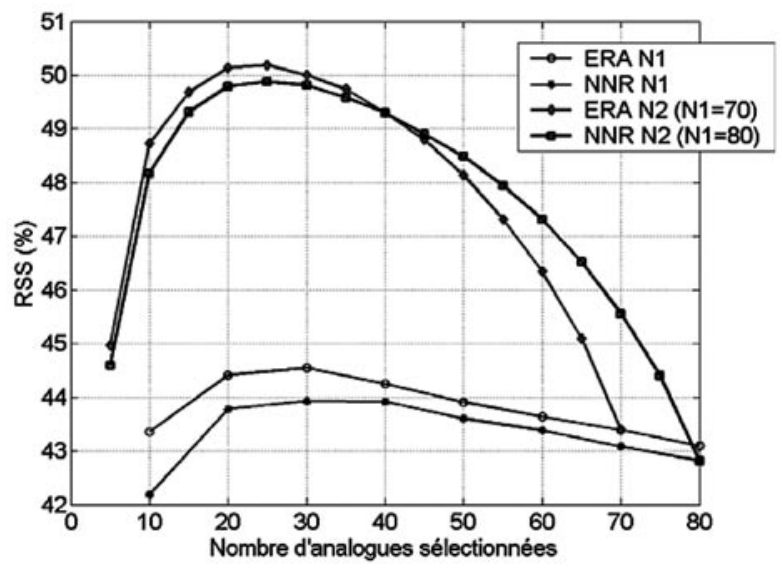

Figure 1 : Evolution du score RSS en fonction du nombre d'analogues sélectionnées : au niveau 1 seul (ERA N1 et NNR N1) et au niveau 2 lorsque N1 est égal au nombre $\mathrm{N} 1$ optimal associé à chaque archive (ERA N2 et NNR N2).

Bien que l'archive ERA contienne des valeurs irréalistes d'humidité, les performances de prévision semblent équivalentes avec les deux archives. Contrairement à ce que l'on aurait pu craindre, les performances au niveau 2 sont même légèrement supérieures avec l'archive ERA. Ce faible gain de performance avec ERA est également obtenu à l'issue du premier niveau d'analogie. Par ailleurs, le niveau 2 d'analogie est bien justifié du fait d'un gain de performances non négligeable apporté par son application (de l'ordre de 5 points de RSS). Ainsi, avec les archives utilisées et dans le cadre de l'application au bassin de la Saône, le nombre d'analogues à sélectionner au second niveau est de 25 . 


\section{III.2 EVALUATION DE LA QUALITÉ DE LA PRÉVISION À L'AIDE DU DIAGRAMME ROC}

Le diagramme ROC (Receiver Operating Characteristic) est utilisé en médecine mais aussi depuis quelques années en météorologie [18]. Ce diagramme trace, pour un seuil $\mathrm{S}-$ ici de pluie - fixé, la probabilité de détection en fonction du taux de fausses détections. La sensibilité de la méthode au choix de l'archive de réanalyses a été étudiée à l'aide de ce diagramme. Pour un seuil donné, les courbes ROC associées aux archives ERA et NNR ont été tracées. En raison du très faible écart entre les deux courbes, nous avons décidé de comparer les aires estimées sous les courbes ROC (score AROC). Ce score vaut 1 pour une prévision parfaite, 0,5 pour une prévision équivalente à la simple prévision climatologique ; et s'il est inférieur à 0,5 , la prévision s'avère de moins bonne qualité que la climatologie.

Cinq seuils de pluie ont été considérés : tous les cumuls de pluie supérieurs à $0 \mathrm{~mm}$, et tous ceux supérieurs aux quantiles de pluie 50, 70, 90 et $99 \%$. Le Tableau 2 présente les scores AROC obtenus pour chaque seuil lorsque chacune des archives est utilisée.

Tableau 2 : Valeurs du score AROC atteint pour différents seuils de pluie lorsque chacune des archives est utilisée

\begin{tabular}{|l|c|c|c|c|c|}
\hline & $\mathbf{0} \mathbf{~ m m}$ & P50 & P70 & P90 & P99 \\
\hline ERA & 0,932 & 0,936 & 0,930 & 0,922 & 0,895 \\
\hline NNR & 0,930 & 0,935 & 0,930 & 0,922 & 0,888 \\
\hline
\end{tabular}

Aucune différence notable n'est observée entre les scores. Cependant, les performances sont légèrement supérieures lorsque l'archive ERA est utilisée, notamment pour les pluies extrêmes (supérieures au quantile P99). L'avantage de ce score est aussi de pouvoir discriminer la prévision entre deux événements : on remarque, lorsque les deux archives sont utilisées, que le score maximal est atteint pour le seuil P50, c'est-à-dire pour les situations dont les cumuls pluviométriques sont compris entre les quantiles P50 et P70 (i.e. pour les journées moyennement pluvieuses).

Supposons à présent qu'en prévision opérationnelle nous disposions des sorties d'un MNP différent du modèle utilisé pour les réanalyses. Pourrait-on chercher dans ces réanalyses des situations analogues à la situation prévue par le MNP sans que les performances ne soient diminuées ? Bien que nous soyons placés dans un contexte de prévision parfaite, nous nous sommes intéressés à l'impact sur les performances de l'utilisation d'une archive, dans laquelle on extrait les situations cibles, différente de celle dans laquelle on extrait les situations analogues. Nous avons ainsi envisagé les quatre cas suivants : situations cibles extraites d'ERA et analogues extraites d'ERA (EE), situations cibles extraites de NNR et analogues extraites de NNR (NN), situations cibles extraites d'ERA et analogues extraites de NNR (EN), situations cibles extraites de NNR et analogues extraites d'ERA
(NE). Le Tableau 3 présente, en considérant chacun des cas, les scores AROC obtenus pour les seuils de pluie considérés précédemment.

Tableau 3 : Valeurs du score AROC atteint pour différents seuils de pluie, en considérant chacun des cas (XY : situations cibles extraites de $\mathrm{X}$ et analogues extraites de $Y$ )

\begin{tabular}{|l|c|c|c|c|c|}
\hline & 0 $\mathbf{~ m m}$ & P50 & P70 & P90 & P99 \\
\hline EE & 0,932 & 0,936 & 0,930 & 0,922 & 0,895 \\
\hline NN & 0,930 & 0,935 & 0,930 & 0,922 & 0,888 \\
\hline EN & 0,929 & 0,933 & 0,927 & 0,922 & 0,891 \\
\hline NE & 0,927 & 0,932 & 0,927 & 0,923 & 0,897 \\
\hline
\end{tabular}

Les résultats montrent qu'en général les performances, bien qu'équivalentes, diminuent légèrement si deux archives différentes sont utilisées. Un gain de performance est cependant obtenu pour les pluies les plus importantes. Ce résultat doit être relativisé compte tenu du faible nombre de valeurs de l'échantillon (110 valeurs). Ainsi si l'on dispose des sorties de plusieurs modèles, cette perspective en mode opérationnel s'avèrerait intéressante dans certaines conditions, par exemple si les sorties de l'un d'entre eux ne sont pas disponibles. Il reste tout de même à effectuer une série de tests via les sorties archivées des MNP opérationnels.

\section{IV — PERSPECTIVES D’AMÉLIORATION}

La partie précédente montre que l'utilisation des deux archives NNR et ERA conduit, dans un contexte de prévision parfaite, à des résultats équivalents pour une résolution spatiale de $2,5^{\circ}$. La prochaine étape est d'exploiter l'archive européenne ERA à sa résolution initiale de $1,125^{\circ}$, ce qui permet d'espérer une meilleure prise en compte des conditions locales lors de l'application du niveau 2. Néanmoins, les premiers résultats montrent que le gain de performance n'est pas significatif.

D'autre part, l'évaluation ne peut avoir un sens que si la prévision se fait dans un contexte opérationnel. Par conséquent, dans le but d'achever l'étude de la sensibilité de la méthode aux archives, des prévisions archivées produites par les modèles GFS et ECMWF seront utilisées pour vérifier l'efficience de la méthode et pour évaluer la réelle aptitude des modèles à prévoir l'évolution des champs des variables d'analogie. Ce travail a déjà débuté dans le cadre d'une étude exploratoire appliquée à la Seine [19].

Parallèlement à ces tests, d'autres perspectives d'amélioration sont envisagées. Actuellement, pour la prévision d'un jour cible, les situations candidates au premier niveau de sélection sont automatiquement sélectionnées dans une fenêtre temporelle de plus ou moins deux mois autour du jour cible. Il n'y a donc aucune souplesse dans cette pré-sélection. Pour remédier à ce problème, il est envisagé de sélectionner les situations candidates selon leur température. Ainsi, un 
certain nombre de situations, proches de la situation cible d'un point de vue thermique, pourrait être retenu. Une autre perspective envisagée est de détecter automatiquement les zones frontales, responsables des épisodes pluvieux sur des grands bassins peu montagneux comme les bassins tests de la Saône et de la Seine. Deux variables a priori pertinentes seront testées : la température potentielle équivalente, qui permet de détecter les changements de masse d'air et les zones d'instabilité atmosphérique ; et la vitesse verticale, qui permet de détecter les zones d'ascendance, responsables des formations nuageuses frontales. L'une de ces variables pourrait ainsi être introduite entre le premier niveau (circulation générale à échelle synoptique) et le second niveau (état hygrométrique de l'atmosphère à échelle locale).

Par ailleurs, la distribution des pluies fournies par les analogues est fondée sur l'équiprobabilité entre les situations. Or, une situation très analogue au niveau 1 ou au niveau 2 pourrait être affectée d'un poids plus important afin de tenir compte de la qualité d'analogie. Une méthode de pondération par les scores obtenus aux niveaux 1 ou 2 pourrait être ainsi envisagée.

Enfin, la méthode de prévision par analogie est utilisée en France principalement sur les bassins à crue rapide à horizon de trois à cinq jours. L'adaptation de la méthode aux bassins à crue lente, sur les bassins tests de la Saône et de la Seine, nécessite de reconsidérer le cumul pluviométrique à prévoir, ce dans le but d'anticiper les événements jusqu'à sept à dix jours à l'avance. Actuellement testée à un pas de temps journalier, il est également prévu de l'adapter à des pas de temps supérieurs pour ce type de bassin.

\section{CONCLUSIONS}

La méthode de prévision des précipitations adaptée par analogie s'est développée ces dernières années en France et a été implémentée dans plusieurs infrastructures. La dernière version, qui décompose la sélection d'analogues en deux niveaux, s'appuie sur une archive de variables d'analogie. Deux archives de réanalyses (ERA-40 et NCEP/NCAR) sont actuellement disponibles à résolution $2,5^{\circ}$ sur des périodes suffisamment longues (de l'ordre d'une quarantaine d'années). La sensibilité de la méthode au choix de l'archive a été étudiée. Les résultats montrent que cette sensibilité est faible ; même si, pour les événements pluvieux extrêmes, les performances de la méthode sont supérieures lorsque l'archive ERA-40 est utilisée.

Plusieurs perspectives d'amélioration de la méthode ont été mentionnées et seront explorées à court terme.

Dans le cas de la prévision des précipitations sur des bassins à crue lente, il faudra adapter la méthode de prévision à l'échelle du temps de réaction de ces bassins, qui est de l'ordre de quelques jours.

\section{Q RÉFÉRENCES}

[1] E. N. Lorenz (1969) - Atmospheric predictability as revealed by natural occurring analogues. J. Atmos. Sci. 26 636-646
[2] G. M. Carter, J. P. Dallavalle, H. R. Glahn (1989) Statistical forecasts based on the National Meteorological Center's numerical weather prediction system. Weather Forecasting. 4 401-412

[3] T. M. Hamill, J. S. Whitaker, S. L. Mullen (2006) Reforecasts : An important dataset for improving weather predictions. Bull. Amer. Meteor. Soc. 87 33-46

[4] G. Bontron (2004) - Prévision quantitative des précipitations : adaptation probabiliste par recherche d'analogues. Utilisation des réanalyses NCEP-NCAR et application aux précipitations du Sud-Est de la France. Thèse de Doctorat, Institut National Polytechnique de Grenoble.

[5] D. Duband (1970) - Reconnaissance dynamique de la forme des situations météorologiques. Application à la prévision quantitative des précipitations. Thèse de $3^{e}$ cycle de la Faculté des Sciences de Paris.

[6] D. Duband (1980) - Dynamic selection of analogue flow patterns to produce quantitative precipitation forecasts. WMO Symposium on probabilistic and statistical method in weather forecasting, Nice, France, 8-12 septembre.

[7] S. Guilbaud (1997) - Prévision quantitative des précipitations journalières par une méthode statistico-dynamique de recherche d'analogues. Thèse de Doctorat, Institut National Polytechnique de Grenoble.

[8] Ch. Obled, G. Bontron, R. Garcon (2002) - Quantitative precipitation forecasts : a statistical adaptation of model outputs through an analogues sorting approach. J. of Atmos. Res. 63 303-324

[9] F. Wetterhall, S. Halldin, C-Y. Xu (2004) - Statistical precipitation downscaling in central Sweden with the analogue method. J. Hydrol. 306 174-190

[10] C. Matulla, X. Zhang, X. Wang, J. Wang, E. Zorita, S. WAGNER, H. VON STORCH (2007) - Influence of similarity measures on the performance of the analog method for downscaling daily precipitation. Clim. Dyn. 30 133-144

[11] V. Altava-Ortiz, A. Barrera, M. C. Llasat, M. A. Prat, J. Gibergans-Bàguena, M. Barnolas (2006) - Application of the MM5 and the analogous method to heavy rainfall event, the case of 16-18 October 2003 in Catalonia (Spain). Adv. in Geosc. 7 313-319

[12] T. Diomede, F. Nerozzi, T. Paccagnella, E. Todini (2008) The use of meteorological analogues to account for LAM QPF uncertainty. Hydrol. Earth Syst. Sci. 12 141-157

[13] E. Kalnay, M. Kanamitsu, R. Kistler, W. Collins, D. Deaven, L. Gandin, M. Iredell, S. Saha, G. White, J. Woollen, Y. Zhu, M. Chelliah, W. Ebisuzaki, W. Higgins, J. Janowiak, K.C. Mo, C. Ropelewski, J. Wang, A. LeetmaA, R. Reynolds, R. Jenne, D. Joseph (1996) — The NCEP/ NCAR 40-Year Reanalysis Project. Bull. Am. Meteorol. Soc. 77 437-471

[14] S.M. Uppala, P.W. Kallberg, A.J. Simmons, U. Andrae, V. Da Costa Bechtold, M. Fiorino, Jk. Gibson, J. Haseler, A. Hernandez, Ga. Kelly, X. Li, K. Onogi, S. SaArinen, N. Sokka, Rp. Allan, E. Andersson, K. Arpe, Ma. Balmaseda, Acm. Beljahr, L. Van De Berg, J. Bidlot, N. Bormann, S. Caires, F. Chevallier, A. Dethof, M. Dragosavac, M. Fisher, M. Fuentes, S. Hagemann, E. Hòlm, Bu. Hoskins, L. Isaksen, Paem. Janssen, R. Jenne, Ap. Mcnally, J-F. Mahfouf, J-J . Morcrette, Na. Rayner, Rw. Saunders, P. Simon, A. Sterl, Ke. Trenberth, A. Untch, 
D. Vasiljevic, P. Viterbo, J. Woollen (2005) — The ERA-40 re-analysis. Q. J. R. Meteorol. Soc. 131 2961-3012

[15] A. Ben Daoud, Ch. Obled, M. Lang, E. Sauquet, G. BONTRON (2008) - Comparison of 850-hPa relative humidity between ERA-40 and NCEP/NCAR re-analyses : detection of suspicious data in ERA. Atmos. Sci. Letters (soumis pour publication). 40

[16] P. Quintana-Segui, P. Le Moigne, Y. Durand, E. Martin, F. Habets, M. Baillon, C. Canellas, L. Franchisteguy, S. Morel (2007) - Analysis of near-surface atmospheric variables : Validation of the SAFRAN analysis over France. $J$. Appl. Meteor. Climatol. 47 92-107
[17] H. Hersbach (2000) - Decomposition of the ranked probability score for ensemble prediction systems. Weather Forecasting. 15 559-570

[18] I. T. Jolliffe, D. B. Stephenson (2003) - Forecast verification. A practitioner's guide in atmospheric science. John Wiley \& Sons Ed.

[19] J. Plasse, A. Ben Daoud (2008) - Application au bassin de la Seine amont d'une méthode de prévision des pluies adaptée par analogie : de la prévision de pluie à la prévision de débit. Rapport technique Cemagref. 